\title{
A VARIATION-OF-CONSTANTS FORMULA FOR A LINEAR ABSTRACT EVOLUTION EQUATION IN HILBERT SPACE
}

\author{
HANZHONG WU ${ }^{1}$
}

(Received 4 January, 2000; revised 23 June, 2000)

\begin{abstract}
A variation-of-constants formula is obtained for a linear abstract evolution equation in Hilbert space with unbounded perturbation and free term. As an application, the state of the abstract controlled system with unbounded mixed controls is explicitly given.
\end{abstract}

\section{Introduction and main results}

In this paper, we consider the following equation in some Hilbert space $X$ :

$$
y(t)=e^{-A t} x+\int_{0}^{t} A^{\alpha} e^{-A(t-s)} \Phi y(s) d s+\int_{0}^{t} A^{\alpha} e^{-A(t-s)} \Psi u(s) d s,
$$

where the parameters in (1.1) satisfy the following hypothesis:

(H) $-A$ generates an analytic semigroup $e^{-A \cdot}$ on $X$ with fractional power $A^{\gamma}$ being well-defined for all $\gamma \in \mathbb{R}, \alpha \in[0,1)$ is fixed, $\Phi \in \mathscr{L}(X), \Psi \in \mathscr{L}(U, X)$ with $U$ being another Hilbert space, $x \in X$ and $u(\cdot) \in L_{l o c}^{1}(0, \infty ; U)$, the set of all locally integrable functions taking values in $U$. When $\alpha=0, e^{-A t}$ is only assumed to be a $C_{0}$-semigroup and is not necessarily analytic. The second and third terms on the right-hand side of (1.1) are called the perturbation and free terms respectively.

In control theory, people sometimes consider the control variable in the form of a state feedback plus another new control variable. The resulting inhomogeneous equation will have not only a perturbation but also a free term. The system will take the form of (1.1), in which $\alpha=0$ corresponds to the bounded control case and $\alpha \in(0,1)$ corresponds to the unbounded control case. For the bounded control case, $[1,6]$ (among others) established a variation-of-constants formula for (1.1).

\footnotetext{
${ }^{1}$ Department of Mathematics, Fudan University, Shanghai 200433, P. R. China; e-mail: hzwu@fudan.edu.cn.

(C) Australian Mathematical Society 2003, Serial-fee code 1446-1811/03
} 
The controlled second-order parabolic equations in a bounded domain $\Omega \subset R^{n}$ can be represented by

$$
y(t)=e^{-A t} x+\int_{0}^{t} A^{\alpha} e^{-A(t-s)} B v(s) d s,
$$

where $B \in \mathscr{L}(U, X)$, and the relevant values of the constant $\alpha$ are as follows (see [4,6], for example): $\alpha=3 / 4+\varepsilon, \forall \varepsilon>0$, for Dirichlet boundary control with $U=L^{2}(\partial \Omega)$, $X=L^{2}(\Omega) ; \alpha=1 / 4+\varepsilon, \forall \varepsilon>0$, for Neumann boundary control with $U=L^{2}(\partial \Omega)$, $X=L^{2}(\Omega)$; and $n / 4<\alpha<1$, for pointwise control with $U=H^{-2 \alpha}(\Omega), X=L^{2}(\Omega)$. Let $v(\cdot)=K y(\cdot)+u(\cdot)$ in (1.2). Then (1.2) becomes (1.1). A natural step is to represent the state $y(\cdot)$ in terms of the new control variable $u(\cdot)$.

In this paper, we will establish a variation-of-constants formula for the linear abstract evolution equation (1.1).

The following notation will be used hereafter: $-A+\Phi A^{\alpha}$ also (see $[3,8]$ ) generates an analytic semigroup $G(\cdot) \triangleq e^{\left(-A+\Phi A^{\alpha}\right)}$. For a given analytic semigroup $e^{-\Theta \cdot}$ with infinitesimal generator $-\Theta$, there exists some $\lambda_{0}>0$ such that $\Theta_{0} \triangleq \lambda_{0} I+\Theta$ admits fractional powers $\Theta_{0}^{\gamma}$ for any $\gamma \in \mathbb{R}$.

Our main result can be stated as follows.

THEOREM. Assume that the hypothesis $(\mathrm{H})$ holds.

(I) The equation

$$
y(t)=e^{-A t} x+\int_{0}^{t} A^{\alpha} e^{-A(t-s)} \Phi y(s) d s
$$

admits a unique solution $y(\cdot) \in C([0, \infty) ; X)$ for any initial condition $x \in X$. Define

$$
S(t) x \triangleq y(t), \quad \forall x \in X, t \in[0, \infty) .
$$

Then $S(\cdot)$ is also an analytic semigroup with infinitesimal generator $-\tilde{A}$ satisfying

$$
\left\{\begin{aligned}
-\tilde{A} & \triangleq A^{\alpha}\left(-A^{1-\alpha}+\Phi\right), \\
D(\widetilde{A}) & \triangleq\left\{x \in D\left(A^{1-\alpha}\right) \mid\left(A^{1-\alpha}-\Phi\right) x \in D\left(A^{\alpha}\right)\right\} .
\end{aligned}\right.
$$

Moreover, it holds that

$$
S(t)=A^{\alpha} G(t) A^{-\alpha}, \quad \forall t \in[0, \infty) .
$$

(II) For any given $\beta \in(\alpha, 1)$, the solution of (1.2) can be expressed as

$$
y(t)=e^{-\tilde{A} t} x+\int_{0}^{t} \tilde{A}_{0}^{\beta} e^{-\bar{A}(t-s)} E(\alpha, \beta) \Psi u(s) d s,
$$

where $E(\alpha, \beta)$ is the extension of $\tilde{A}_{0}^{-\beta} A^{\alpha}$ on $X$. 
In the above, (1.7) is called a variation-of-constants formula for the abstract evolution equation (1.1).

The remainder of this paper is organized as follows. In Section 2 we prove (I) while in Section 3 we prove (II).

\section{Some linear homogeneous equations}

In this section, we will establish the unique existence of and obtain regularity properties for the solution of some homogeneous equations. These results will be later used to prove (I).

For any $\gamma>0$, there exists a $c_{\gamma}>0$ such that (see [8])

$$
\left\|A^{\gamma} e^{-A t}\right\| \leq c_{\gamma} t^{-\gamma}, \quad \forall t>0
$$

All the above properties also hold for the adjoint $A^{*}$ of $A$.

LEMMA 2.1. It holds that

$$
D\left(A^{\gamma+\varepsilon}\right) \subset D\left(\left[A-\Phi A^{\alpha}\right]_{0}^{\gamma}\right) \subset D\left(A^{\gamma-\varepsilon}\right), \quad \forall \gamma \in[0,1), \varepsilon>0
$$

For any given $\beta \in(\alpha, 1)$, there exists a $d_{\beta}>0$ such that

$$
\left\|A^{\alpha} G(t)\right\| \leq d_{\beta} t^{-\beta}, \quad \forall t>0 .
$$

Proof. From a corollary of Lemma 2.1 in [5], (2.2) and (2.3) follow.

LEMMA 2.2. Suppose that $S_{1}(\cdot)=e^{A_{1} \cdot}$ and $S_{2}(\cdot)=e^{A_{2} \cdot}$ are $C_{0}$-semigroups. If $A_{1} \subseteq A_{2}$, then $A_{1}=A_{2}$ and $S_{1}(\cdot)=S_{2}(\cdot)$.

Proof. By the Hille-Yosida theorem, we have from $A_{1} \subseteq A_{2}$ that there exists a $\lambda_{*}>0$ such that, for $\operatorname{Re} \lambda \geq \lambda_{*}$,

$$
\left(\lambda I-A_{1}\right)^{-1}=\left(\lambda I-A_{2}\right)^{-1} .
$$

Therefore Corollary 7.5 in $\left[8\right.$, p. 29] implies that $A_{1}$ and $A_{2}$ generate the same $C_{0^{-}}$ semigroup.

LEMMA 2.3. For any given $\xi \in X$,

$$
z(t)=e^{-A t} \xi+\int_{0}^{t} e^{-A(t-\tau)} \Phi A^{\alpha} z(\tau) d \tau, \quad t \geq 0,
$$


admits a unique solution $z(\cdot)=G(\cdot) \xi$. Moreover,

$$
\begin{aligned}
G(t-s) \xi & =e^{-A(t-s)} \xi+\int_{s}^{t} e^{-A(t-r)} \Phi A^{\alpha} G(r-s) \xi d r \\
& =e^{-A(t-s)} \xi+\int_{s}^{t} G(t-r) \Phi A^{\alpha} e^{-A(r-s)} \xi d r, \quad \forall 0 \leq s \leq t .
\end{aligned}
$$

ProoF. Define $T \triangleq\left((1-\alpha) / 2 c_{\alpha}\|\Phi\|\right)^{\alpha-1}$ and

$$
F: L^{1}\left(0, T ; D\left(A^{\alpha}\right)\right) \rightarrow L^{1}\left(0, T ; D\left(A^{\alpha}\right)\right)
$$

as follows:

$$
[F f(\cdot)](\cdot) \triangleq e^{-A \cdot \xi}+\int_{0}^{\cdot} e^{-A(\cdot-s)} \Phi A^{\alpha} f(s) d s, \quad \forall f(\cdot) \in L^{1}\left(0, T ; D\left(A^{\alpha}\right)\right) .
$$

It follows from (2.1) and the Fubini theorem that

$$
\begin{aligned}
& \left\|F f_{1}(\cdot)-F f_{2}(\cdot)\right\|_{L^{\prime}\left(0, T: D\left(A^{\alpha}\right)\right.} \\
& \quad \leq c_{\alpha}\|\Phi\| \int_{0}^{T} \int_{0}^{t} \frac{\left\|f_{1}(s)-f_{2}(s)\right\|_{D\left(A^{\alpha}\right)}}{|t-s|^{\alpha}} d s d t \\
& \quad=c_{\alpha}\|\Phi\| \int_{0}^{T} \int_{s}^{T} \frac{1}{|t-s|^{\alpha}} d t\left\|f_{1}(s)-f_{2}(s)\right\|_{D\left(A^{\alpha}\right)} d s \\
& \quad \leq \frac{1}{2}\left\|f_{1}(\cdot)-f_{2}(\cdot)\right\|_{L^{\prime}\left(0, T ; D\left(A^{\alpha}\right)\right.}, \quad \forall f_{1}(\cdot), f_{2}(\cdot) \in L^{1}\left(0, T ; D\left(A^{\alpha}\right)\right) .
\end{aligned}
$$

The contraction mapping principle implies that (2.5) admits a unique solution $z(\cdot ; \xi) \in C([0, T] ; X)$. Applying this principle in the same manner to $[T, 2 T]$, $[2 T, 3 T]$, and so forth, we have that $(2.5)$ admits a unique solution $z(\cdot ; \xi) \in$ $C([0, \infty) ; X)$. Define the operators $Z(t)$ as follows:

$$
Z(t) \xi \triangleq z(t ; \xi), \quad \forall \xi \in X, t \in[0, \infty) .
$$

Then $Z(\cdot)$ satisfies the semigroup property. By (2.1), it holds that, for all $t \geq 0$,

$$
\|z(t ; \xi)\|_{D\left(A^{\alpha}\right)} \leq c_{\alpha} t^{-\alpha}\|\xi\|+c_{\alpha}\|\Phi\| \int_{0}^{t}(t-\tau)^{-\alpha}\|z(\tau ; \xi)\|_{D\left(A^{\alpha}\right)} d \tau .
$$

Thus, by the singular Gronwall inequality ([2, Lemma 7.1.1]), we obtain $Z(t) \in \mathscr{L}(X)$ for $t \in(0, \infty)$, which implies that $Z(\cdot)$ is a $C_{0}$-semigroup.

On the other hand, for any given $\xi \in D\left(A^{\alpha}\right)$, it follows from (2.5) that, for all $t \geq 0$,

$$
\|z(t ; \xi)\|_{D\left(A^{a}\right)} \leq\left\|e^{-A t}\right\|\|\xi\|_{D\left(A^{a}\right)}+c_{\alpha}\|\Phi\| \int_{0}^{t}(t-\tau)^{-\alpha}\|z(\tau ; \xi)\|_{D\left(A^{a}\right)} d \tau
$$


The singular Gronwall inequality and (2.5) implies that $z(\cdot ; \xi) \in C\left([0, \infty) ; D\left(A^{\alpha}\right)\right)$. Furthermore, for any $\xi \in D\left(-A+\Phi A^{\alpha}\right)(=D(A)),(2.5)$ yields that

$$
\lim _{t \rightarrow 0+} \frac{Z(t) \xi-\xi}{t}=-A \xi+\Phi A^{\alpha} \xi
$$

Thus we have from Lemma 2.2 that $z(\cdot ; \xi)=G(\cdot) \xi$.

Next, let $s$ be fixed and define

$$
w(t) \triangleq e^{-A(t-s)} \xi+\int_{s}^{t} G(t-r) \Phi A^{\alpha} e^{-A(r-s)} \xi d r .
$$

By the Fubini theorem and (2.5), it holds that

$$
\begin{aligned}
& \int_{s}^{t} e^{-A(t-r)} \Phi A^{\alpha} w(r) d r \\
&=\int_{s}^{t} e^{-A(t-r)} \Phi A^{\alpha} e^{-A(r-s)} \xi d r \\
&+\int_{s}^{t} e^{-A(t-r)} \Phi A^{\alpha} \int_{s}^{r} G(r-\sigma) \Phi A^{\alpha} e^{-A(\sigma-s)} \xi d \sigma d r \\
&= \int_{s}^{t} e^{-A(t-r)} \Phi A^{\alpha} e^{-A(r-s)} \xi d r \\
&+\int_{s}^{t} \int_{\sigma}^{t} e^{-A(t-r)} \Phi\left[A^{\alpha} G(r-\sigma)\right] \Phi A^{\alpha} e^{-A(\sigma-s)} \xi d r d \sigma \\
&= \int_{s}^{t} e^{-A(t-r)} \Phi A^{\alpha} e^{-A(r-s)} \xi d r \\
&+\int_{s}^{t}\left[G(t-\sigma)-e^{-A(t-\sigma)}\right] \Phi A^{\alpha} e^{-A(\sigma-s)} \xi d \sigma \\
&= \int_{s}^{t} G(t-\sigma) \Phi A^{\alpha} e^{-A(\sigma-s)} \xi d \sigma=w(t)-e^{-A(t-s)} \xi
\end{aligned}
$$

Then the uniqueness of the solution to (2.5) yields (2.6).

Now we will prove the analyticity of $S(\cdot)$ in (1.4).

LEMMA 2.4. For any initial condition $x \in X$, (1.3) admits a unique solution $y(\cdot) \in$ $C([0, \infty) ; X)$. Moreover, $S(\cdot)$ in (1.4) is an analytic semigroup with infinitesimal generator $-\tilde{A}$ satisfying (1.5).

Proof. We can easily have from (2.1) and the singular Gronwall inequality that (1.3) admits a unique solution $y(\cdot) \in C([0, \infty) ; X)$. On the other hand (see $[5$, Theorem 2.2]), $S(t)$ is a $C_{0}$-semigroup with infinitesimal generator $-\tilde{A}$ satisfying (1.5). 
By the perturbation theory of analytic semigroups ( see $[3,8]$ ),

$$
A_{1} \triangleq-A^{*}+\Phi^{*}\left(A^{*}\right)^{\alpha}
$$

also generates an analytic semigroup. In addition, $D\left(A_{1}\right)=D\left(A^{*}\right)$.

By (1.5), it holds that

$$
\langle-\widetilde{A} x, y\rangle=\left\langle x, A_{1} y\right\rangle, \quad \forall x \in D(\widetilde{A}), y \in D\left(A_{1}\right) .
$$

Thus $A_{1} \subseteq(-\widetilde{A})^{*}$. It follows from Lemma 2.2 that $A_{1}=(-\widetilde{A})^{*}$ and $-\tilde{A}$ generates an analytic semigroup.

PrOOF OF (I). Combining Theorem 2.2 in [5] and Lemma 2.4 yields (I).

\section{A variation-of-constants formula}

In this section, we will prove (II) so as to establish a variation-of-constants formula for the evolution equation (1.1).

LEMMA 3.1. For any initial condition $\xi$,

$$
z(t)=e^{-A t} \xi+\int_{0}^{t} e^{-A(t-s)} \Phi A^{\alpha} z(s) d s+\int_{0}^{t} e^{-A(t-s)} \Psi u(s) d s
$$

admits a unique solution $z(\cdot) \in L_{l o c}^{1}\left[0, \infty ; D\left(A^{\alpha}\right)\right)$. Moreover, the solution can be represented by

$$
z(t)=G(t) \xi+\int_{0}^{t} G(t-s) \Psi u(s) d s, \quad \forall t \in[0, \infty)
$$

PROof. The first part of the lemma can be proved similarly to Lemma 2.3. Define

$$
\bar{z}(t) \triangleq G(t) \xi+\int_{0}^{t} G(t-s) \Psi u(s) d s, \quad \forall t \in[0, \infty)
$$

By (2.1) and Lemma 2.1, we have that $\bar{z}(\cdot) \in L_{\mathrm{loc}}^{1}\left[0, \infty ; D\left(A^{\alpha}\right)\right.$ ). Therefore (3.3), Lemma 2.3 and the Fubini theorem imply that

$$
\begin{aligned}
\int_{0}^{t} e^{-A(t-s)} \Phi A^{\alpha} \bar{z}(s) d s= & \int_{0}^{t} e^{-A(t-s)} \Phi A^{\alpha} G(s) \xi d s \\
& +\int_{0}^{t} \int_{r}^{t} e^{-A(t-s)} \Phi A^{\alpha} G(s-r) \Psi u(r) d s d r \\
= & {\left[G(t)-e^{-A t}\right] \xi+\int_{0}^{t}\left[G(t-r)-e^{-A(t-r)}\right] \Psi u(r) d r } \\
= & \bar{z}(t)-e^{-A t} \xi-\int_{0}^{t} e^{-A(t-s)} \Psi u(s) d s
\end{aligned}
$$

By the uniqueness of the solution to (3.1), (3.2) follows from (3.4). 
LEMMA 3.2. For any given $\beta \in(\alpha, 1)$, there exists some $C_{\beta}>0$ such that

$$
\left\|\tilde{A}_{0}^{-\beta} A^{\alpha} x\right\| \leq C_{\beta}\|x\|, \quad \forall x \in D\left(A^{\alpha}\right) .
$$

Denote by $E(\alpha, \beta)$ the extension of the operator $\widetilde{A}_{0}^{-\beta} A^{\alpha}$ on $X$. Then it holds that

$$
A^{\alpha} G(t)=\widetilde{A}_{0}^{\beta} A^{\alpha} G(t) A^{-\alpha} E(\alpha, \beta), \quad \forall t \in(0, \infty) .
$$

PROOF. It has been proved from the proof of Lemma 2.4 that $\widetilde{A}^{*}=A^{*}-\Phi^{*} A^{* \alpha}$. Lemma 2.1 in [5] implies $A^{* \alpha} \tilde{A}_{0}^{-\beta} \in \mathscr{L}(X)$. Obviously

$$
\left\langle\widetilde{A}_{0}^{-\beta} A^{\alpha} x, y\right\rangle=\left\langle x, A^{* \alpha}\left[A^{*}-\Phi^{*} A^{* \alpha}\right]_{0}^{-\beta} y\right\rangle, \quad \forall x \in D\left(A^{\alpha}\right), y \in X,
$$

which yields (3.5). By (1.5) and (1.6), it holds that

$$
\begin{aligned}
A^{\alpha} G(t) x & =A^{\alpha} G(t) A^{-\alpha} \tilde{A}_{0}^{\beta} \widetilde{A}_{0}^{-\beta} A^{\alpha} x=S(t) \widetilde{A}_{0}^{\beta} E(\alpha, \beta) x \\
& =\widetilde{A}_{0}^{\beta} S(t) E(\alpha, \beta) x, \quad \forall x \in D\left(A^{\alpha}\right), t \in(0, \infty) .
\end{aligned}
$$

Due to the density of $x \in D\left(A^{\alpha}\right)$ in $X,(3.6)$ follows from (3.8).

Proof OF (II). For any given $x \in X$, let $\xi=A^{-\alpha} x$. Lemma 3.1 tells us that (3.1) admits a unique solution $z(\cdot) \in L_{\text {loc }}^{1}\left[0, \infty ; D\left(A^{\alpha}\right)\right)$. Thus $A^{\alpha} z(\cdot)$ is the unique solution to (1.1). Combining (1.6), (3.2) and (3.6) yields (II).

\section{Acknowledgements}

This work has been partially supported by the National Key Project of China, the National Nature Science Foundation of China No. 19901030 and the Laboratory of Mathematics for Nonlinear Sciences at Fudan University.

\section{References}

[1] R. Curtain and A. Pritchard, "The infinite-dimensional Riccati equation for systems defined by evolution operators", SIAM J. Control Optim. 14 (1976) 951-983.

[2] D. Henry, Geometric theory of semilinear parabolic equations (Springer, New York, 1981).

[3] T. Kato, Perturbation theory for linear operators (Springer, New York, 1980).

[4] I. Lasiecka, "Unified theory for abstract parabolic boundary problem: a semigroup approach", Appl. Math. Optim. 6 (1980) 283-333.

[5] X. Li and K. Liu, "The effect of small time delays in the feedbacks on boundary stabilization", Science in China, Ser. A 36 (1993) 1435-1443.

[6] X. Li and J. Yong, Optimal control theory for infinite dimensional systems (Birkhäuser, Boston, 1995). 
[7] J. L. Lions, Optimal control of systems governed by partial differential equations (Springer, New York, 1971).

[8] A. Pazy, Semigroups of linear operators and applications to partial differential equations (Springer, New York, 1983).

[9] H. Wu, "Some equivalent conditions for exponential stabilization of linear systems with unbounded control", Science in China, Ser. E 42 (1999) 252-259. 